\title{
Enhanced Medial Prefrontal-Default Mode Network Functional Connectivity in Chronic Pain and Its Association with Pain Rumination
}

\author{
Aaron Kucyi, ${ }^{1,2,8}$ Massieh Moayedi, ${ }^{1,2,6,8}$ Irit Weissman-Fogel, ${ }^{1,7,8}$ Michael B. Goldberg, ${ }^{3,4,8}$ Bruce V. Freeman, ${ }^{3,4}$ \\ Howard C. Tenenbaum, ${ }^{1,3,4}$ and Karen D. Davis ${ }^{1,2,5,8}$ \\ ${ }^{1}$ Division of Brain, Imaging and Behaviour-Systems Neuroscience, Toronto Western Research Institute, University Health Network, Toronto, Ontario, \\ Canada M5T 2S8, ${ }^{2}$ Institute of Medical Science, University of Toronto, Toronto, Ontario, Canada M5S 1A8, ${ }^{3}$ Faculty of Dentistry, University of Toronto, \\ Toronto, Ontario, Canada M5G 1G6, ${ }^{4}$ Mount Sinai Hospital Dental Clinic, Toronto, Ontario, Canada M5G 1X5, ${ }^{5}$ Department of Surgery, University of \\ Toronto, Toronto, Ontario, Canada M5T 1P5, 'Department of Neuroscience, Physiology and Pharmacology, University College London, United Kingdom \\ WC1E 6BT, ${ }^{7}$ Department of Physical Therapy, Faculty of Social Welfare and Health Sciences, University of Haifa, Haifa, Israel 3498838, and ${ }^{8}$ University of \\ Toronto Centre for the Study of Pain, Toronto, Ontario, Canada M5T 1P8
}

Rumination is a form of thought characterized by repetitive focus on discomforting emotions or stimuli. In chronic pain disorders, rumination can impede treatment efficacy. The brain mechanisms underlying rumination about chronic pain are not understood. Interestingly, a link between rumination and functional connectivity (FC) of the brain's default mode network (DMN) has been identified within the context of mood disorders. We, and others, have also found DMN dysfunction in chronic pain populations. The medial prefrontal cortex (mPFC) is a key node of the DMN that is anatomically connected with the descending pain modulatory system. Therefore, we tested the hypothesis that in patients with chronic pain, the mPFC exhibits abnormal FC related to the patient's degree of rumination about their pain. Seventeen patients with idiopathic temporomandibular disorder (TMD) and 17 age- and sex-matched healthy controls underwent resting state functional MRI, and rumination about pain was assessed through the rumination subscale of the Pain Catastrophizing Scale. Compared with healthy controls, we found that TMD patients exhibited enhanced mPFC FC with other DMN regions, including the posterior cingulate cortex $(\mathrm{PCC})$ /precuneus $(\mathrm{PCu})$ and retrosplenial cortex. We also found that individual differences in pain rumination in the chronic pain patients (but not in healthy controls) were positively correlated to mPFC FC with the $\mathrm{PCC} / \mathrm{PCu}$, retrosplenial cortex, medial thalamus, and periaqueductal/periventricular gray. These data implicate communication within the DMN and of the DMN with the descending modulatory system as a mechanism underlying the degree to which patients ruminate about their chronic pain.

Key words: connectivity; default mode; medial prefrontal cortex; pain; rumination; TMD

\section{Introduction}

Pain rumination, or perseverative negative thinking about pain and its possible causes and consequences, has been linked with higher pain levels and poorer clinical outcomes in multiple

\footnotetext{
Received Dec. 3, 2013; revised Jan. 14, 2014; accepted Feb. 8, 2014.

Author contributions: A.K., M.M., I.W.-F., M.B.G., B.V.F., H.C.T., and K.D.D. designed research; M.M. and I.W.-F. performed research; A.K. and M.M. analyzed data; A.K., M.M., I.W.-F., and K.D.D. wrote the paper.

This study was funded by the Canadian Institute of Health Research (CIHR) Grant and funds from the Canada Research Chair program (MOP 53304 to K.D.D.), CIHR studentship (M.M. and A.K.); Ontario Graduate Scholarship (M.M. and A.K.), CIHR Strategic Training programs: Pain: Molecules to Community (M.M. and I.W.-F.), and Cell Signals in Mucosal Inflammation and Pain (STP-53877 to M.M.), University of Toronto Centre for the Study of Pain Clinician/Scientist Research fellowship (I.W.-F.). This work was performed at Toronto Western Research Institute, University Health Network. We thank Eugen Hlasny and Keith Ta for expert technical assistance, Dr Yair Lenga for patient screening, and Dr Tim Salomons for feedback on the analyses.

The authors declare no competing financial interests.

Correspondence should be addressed to Dr Karen D. Davis, Toronto Western Research Institute, Division of Brain Imaging and Behaviour, Systems Neuroscience, 399 Bathurst Street, Room MP14-306, Toronto, Ontario, Canada M5T 2S8.E-mail: kdavis@uhnres.utoronto.ca.

DOI:10.1523/JNEUROSCI.5055-13.2014

Copyright $\odot 2014$ the authors $\quad 0270-6474 / 14 / 343969-07 \$ 15.00 / 0$
}

chronic pain populations (Sullivan et al., 2002; Van Damme et al., 2002; Buenaver et al., 2012). Pain rumination is similar to but partially distinct from emotionally negative thinking patterns that occur in psychiatric disorders, such as depression (NolenHoeksema, 2000; Sansone and Sansone, 2012). Whereas rumination always involves excessive attention to distressing thoughts and stimuli, the specific focus on pain could uniquely affect brain networks associated with attention, pain perception, and pain modulation.

There is a link between rumination and the brain's default mode network (DMN) within the context of mood in healthy individuals and in major depressive disorder patients (Kross et al., 2009; Zhu et al., 2012). The DMN is active when people are not engaged with any specific task or stimulus but is suppressed when attention shifts to a task or stimulus (Raichle and Snyder, 2007). DMN regions, including the medial prefrontal cortex (mPFC), posterior cingulate cortex (PCC), and lateral parietal cortices, exhibit functional connectivity (FC) during a stimuli-/ task-independent ("resting") state and during task states in that 
their activity fluctuations are correlated with one another (Greicius et al., 2003). DMN activation increases correspond to attention lapses (Weissman et al., 2006), autobiographical recall/planning (Andrews-Hanna et al., 2010), and mindwandering (Christoff et al., 2009; Kucyi et al., 2013), suggesting that this network supports attention toward introspective thoughts.

Acute pain, an intrinsically salient experience, would be expected to deactivate the DMN. However, there are individual differences in tendencies to attend to pain and increases in DMN activity during routine mind-wandering away from pain (Kucyi et al., 2013). In chronic pain, rumination about ongoing pain may be represented in FC within the DMN or between the DMN and pain-related networks. Several studies have reported aberrant DMN FC in chronic pain (Baliki et al., 2008, 2011; Napadow et al., 2010; Loggia et al., 2013), but it is unknown whether these abnormalities relate to pain rumination.

Chronic pain populations also exhibit brain abnormalities in the descending pain modulatory system, most notably in the periaqueductal gray (PAG), and this may relate to dysfunctional pain regulation (Rocca et al., 2006; Mainero et al., 2011; Desouza et al., 2013). We demonstrated that in healthy individuals, DMNPAG FC is enhanced during attentional fluctuations away from pain (Kucyi et al., 2013). Furthermore, mPFC-PAG structural and functional connectivity relate to individual differences in tendencies to attend to pain. Therefore, the mPFC is well positioned to mediate changes of functional interactions between the $\mathrm{DMN}$ and descending pain modulatory system in chronic pain, possibly driven by pain rumination.

Here we tested the hypothesis that the MPFC exhibits abnormal resting state FC in chronic pain and that this abnormality relates to a patient's degree of pain rumination. We first compared $\mathrm{mPFC} F \mathrm{~F}$ in patients with idiopathic temporomandibular disorder with that of age-matched healthy individuals. We then related $\mathrm{mPFC} F \mathrm{~F}$ with individual differences in pain rumination.

\section{Materials and Methods}

Participants. Seventeen right-handed females with idiopathic temporomandibular disorder (TMD; mean age \pm SD, $33.1 \pm 11.9$ years) and 17 age-matched healthy right-handed females (mean age \pm SD, $32.2 \pm 10.2$ years) were recruited and provided informed written consent for procedures approved by the University Health Network and Mount Sinai Hospital Research Ethics Boards. Data from the same cohort regarding brain structural and functional abnormalities, that are distinct from the data used in this study, were previously reported (Moayedi et al., 2011, 2012a,b; Weissman-Fogel et al., 2011; Salomons et al., 2012). Dentists, who are TMD specialists in the Pain Unit of the Mount Sinai Hospital Dental Clinic, examined and diagnosed patients as having TMD using standard clinical diagnostic criteria from this institution. Criteria included the involvement of myofascial and/or temporomandibular joint, based on clinical examination. Inclusion criteria included: (1) nontraumatic TMD; (2) musculoligamentous pain in the temporomandibular area; (3) pain in the muscles of mastication rated verbally as at least $4 / 10$ for at least 3 months at the time of evaluation, or pain that is aggravated by mandibular function; and (4) moderate ( $\geq 2$ of 4 -point Likert scale of $0=$ "no pain" to $3=$ "severe pain" to palpation and/or pain persisting postexamination in at least three muscle sites and/or moderate pain to palpation of the temporomandibular joint and/or limited mandibular movement (opening $<40 \mathrm{~mm}$ ). Exclusion criteria for all subjects included: (1) left-handedness; (2) self-report of metabolic, rheumatoid or vascular diseases/disorders, or any other serious diseases; (3) self-report of commonly comorbid functional chronic pain disorders (irritable bowel syndrome and fibromyalgia); (4) self-report of psychiatric disorders (e.g., depression, schizophrenia); (5) self-report history of an abnormal neurological examination; (6) contraindication to MRI scanning; and (7) self-report of substance abuse. Additionally, healthy controls were excluded if they had a history of chronic pain. Patients verbally reported their average pain intensity over the last month on a scale from 0 to $10(0=$ "no pain," $10=$ "the worst pain imaginable"; mean $=4.3$, $\mathrm{SD}=1.8)$

Assessment of pain rumination. Pain rumination was assessed with the rumination subscale of the Pain Catastrophizing Scale (PCS; Sullivan et al., 1995), a well validated measure of maladaptive thinking patterns related to pain. Subjects completed the full PCS, which consists of 13 items measured on a five-point Likert scale $(0=$ "not at all," $4=$ "all the time"). The rumination subscale consists of four items measuring the degree to which individuals focus on their pain (sample item: "I can't stop thinking about how much it hurts"). A two-tailed independent samples $t$ test revealed no significant differences in pain rumination scores between patients $($ mean $=6.8, \mathrm{SD}=3.94)$ and controls $($ mean $=$ $6.4, \mathrm{SD}=4.06 ; p=0.77$ ). Within the patient group, there was no significant correlation between pain rumination and average pain intensity $(r=-0.26, p=0.31)$.

MRI acquisition. Brain imaging was performed with a 3T GE Signa HDx MRI system fitted with an eight-channel phased array head coil. Before the resting state BOLD fMRI scan, subjects were given the following instructions: "Close your eyes, stay calm, and do not think of anything in particular. Do not fall asleep." Scans were then acquired during a 5 min $8 \mathrm{~s}$ period using $\mathrm{T} 2^{\star}$-weighted echo-planar imaging $(\mathrm{TR}=2000$ $\mathrm{ms}, \mathrm{TE}=40 \mathrm{~ms}$, flip angle $=45^{\circ} ; 28$ axial slices per volume, $64 \times 64$ matrix, $3.125 \times 3.125 \times 4 \mathrm{~mm}^{3}$ voxels). High-resolution whole brain T1-weighted anatomical scans were also acquired with a threedimensional IR-FSPGR sequence $(\mathrm{TR}=12 \mathrm{~ms}, \mathrm{TE}=5 \mathrm{~ms}$, TI $=300 \mathrm{~ms}$, flip angle $=20^{\circ}$; 128 axial slices, $256 \times 256$ matrix, field-of-view $24 \times 24$ $\mathrm{cm}, 0.94 \times 0.94 \times 1.5 \mathrm{~mm}^{3}$ voxels).

Data preprocessing. Preprocessing procedures were performed with FSL v5.0 (Jenkinson et al., 2012), MATLAB v7.12.0 (MathWorks), and fMRISTAT (Worsley et al., 2002), and were in line with our previous studies (Kucyi et al., 2012, 2013). The following steps were first performed using FSL's FEAT: deletion of the first four volumes, motion correction (MCFLIRT), brain extraction (BET), and linear registration (FLIRT) among fMRI, T1-weighted anatomical, and standard MNI152 space $\left(2 \mathrm{~mm}^{3}\right.$ resolution) images. To remove physiological/scannerrelated noise from $\mathrm{fMRI}$ data, we implemented aCompCor procedures (Behzadi et al., 2007; Chai et al., 2012). The T1-weighted images were segmented into gray matter, white matter (WM), and CSF using FSL's FAST. The WM and CSF partial volume estimate maps were transformed to fMRI space using the previously computed linear transform. These maps were thresholded to retain only the top $198 \mathrm{~cm}^{3}$ (WM) and top $20 \mathrm{~cm}^{3}$ (CSF) with highest probability of being a given tissue type (Chai et al., 2012) to avoid the inclusion of voxels with partial volumes of different tissue types. The thresholded images were then multiplied by the 4-D data, and principal components analysis was performed separately within WM and CSF. The top five WM components, top five CSF components, and six motion parameters obtained with MCFLIRT were regressed out of the fMRI data. Spatial smoothing $(6 \mathrm{~mm}$ full-width half-maximum kernel) and bandpass temporal filtering $(0.01-0.1 \mathrm{~Hz})$ were then performed.

mPFC seed definition. The seed region was based on peak MNI coordinates from our previous study in which we found greater $\mathrm{mPFC}$ activation (along with other DMN regions) during attention away from pain compared with attention toward pain (Kucyi et al., 2013). A 6-mmdiameter sphere was drawn surrounding these reported $\mathrm{mPFC}$ coordinates $(x y z=-2,58,-6$; Fig. $1 A)$. The standard space mPFC image was linearly transformed to fMRI space, and the mean time course across all voxels in the seed was extracted.

Statistical analyses. A first-level general linear model (GLM) was performed within-subjects using FEAT with FILM prewhitening. The mean mPFC time course was entered as a regressor to identify voxels throughout the whole brain that were functionally connected with this seed. Resulting statistical images were entered into a second (group-level) mixed-effects GLM using FLAME $1+2$. Statistical contrasts were performed to identify (1) mPFC FC within the healthy control group, (2) mPFC FC within the TMD group, (3) regions with greater mPFC FC for 


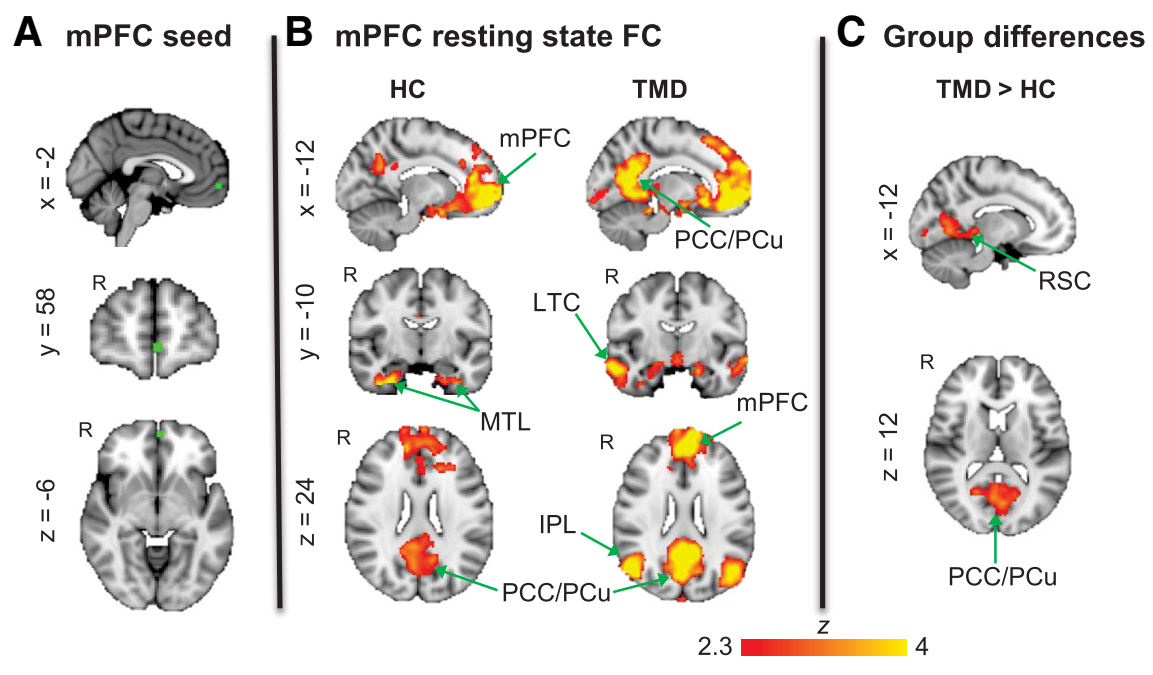

Figure 1. $\mathrm{mPFC} F$ in healthy controls versus TMD patients. $\boldsymbol{A}$, Location of the $\mathrm{mPFC}$ seed region in standard MNI152 space. $\boldsymbol{B}$, Brain regions exhibiting resting state $\mathrm{FC}$ with the $\mathrm{mPFC}$ in healthy controls $(\mathrm{HC})(n=17)$ and TMD patients $(n=17)$. C, Brain regions exhibiting enhanced resting state $F C$ with the $\mathrm{MPFC}$ in TMD patients compared with healthy controls. All statistical images are thresholded at whole-brain FWE-corrected Z $>2.3$; cluster-based $p<0.05$. LTC, Lateral temporal cortex; MTL, medial temporal lobe; FC, functional connectivity; $\mathrm{HC}$, healthy controls.

Table 1. Peak MNI coordinates for regions with significant mPFC resting state functional connectivity in TMD patients and healthy controls, and significantly enhanced $\mathrm{mPFC}$ functional connectivity in TMD patients compared with healthy controls (FWE-corrected at $Z>2.3$, cluster-based threshold of $p<0.05$ )

\begin{tabular}{|c|c|c|c|c|}
\hline \multirow[b]{2}{*}{ Region } & \multirow[b]{2}{*}{ Z-max } & \multicolumn{3}{|c|}{ Peak voxel (MNI coordinates, mm) } \\
\hline & & $x$ & $y$ & $z$ \\
\hline \multirow{2}{*}{\multicolumn{5}{|c|}{$\begin{array}{l}\text { mPFC functional connectivity } \\
\quad \text { Controls }\end{array}$}} \\
\hline & & & & \\
\hline $\mathrm{mPFC}$ & 9.0 & 0 & 58 & -8 \\
\hline Rprecuneus & 3.66 & 8 & -64 & 36 \\
\hline R parahippocampal gyrus & 4.5 & 30 & -10 & -34 \\
\hline R middle frontal gyrus & 3.38 & 26 & 32 & 34 \\
\hline L parahippocampal gyrus & 3.17 & -34 & -10 & -34 \\
\hline R superior frontal gyrus & 2.82 & 28 & 36 & 54 \\
\hline $\mathrm{PCC}$ & 2.74 & 2 & -14 & 30 \\
\hline L superior frontal gyrus & 2.74 & -4 & 32 & 62 \\
\hline R temporal pole & 2.69 & 50 & 8 & -30 \\
\hline Retrosplenial cortex & 2.72 & -8 & -52 & 8 \\
\hline \multicolumn{5}{|l|}{ TMD } \\
\hline $\mathrm{mPFC}$ & 7.72 & -2 & 58 & -4 \\
\hline L inferior parietal lobule & 4.76 & -38 & -78 & 36 \\
\hline R inferior parietal lobule & 5.12 & 50 & -68 & 26 \\
\hline R lateral temporal cortex & 4.69 & 60 & -12 & -20 \\
\hline L lateral temporal cortex & 4.15 & -64 & -18 & -14 \\
\hline Visual cortex & 3.66 & 4 & -90 & 2 \\
\hline R orbitofrontal cortex & 3.98 & 36 & 32 & -18 \\
\hline L posterior thalamus & 3.65 & -8 & -30 & -2 \\
\hline R parahippocampal gyrus & 2.86 & 12 & 4 & -20 \\
\hline R orbitofrontal cortex & 2.55 & -18 & 22 & -22 \\
\hline \multicolumn{5}{|l|}{ TMD $>$ controls } \\
\hline Lingual gyrus & 3.74 & 6 & -66 & 6 \\
\hline Occipital pole & 3.17 & -2 & -88 & -2 \\
\hline R PCC/precuneus (ventral) & 3.15 & 22 & -62 & 12 \\
\hline Precuneus (dorsal) & 3.11 & 0 & -78 & 42 \\
\hline R retrosplenial cortex & 3.07 & 8 & -50 & -8 \\
\hline
\end{tabular}

The image was thresholded at $Z>3$ to obtain peak coordinates.

controls compared with patients, and (4) regions with greater mPFC for patients compared with controls. All contrasts were thresholded at the whole-brain FWE-corrected level $(Z>2.3$; cluster $p<0.05)$.

In addition to the analysis of group differences, we conducted a mixedeffects group-level GLM (FLAME $1+2$ ) within the patient group. Here we entered demeaned pain rumination scores as a regressor to identify brain regions with either positive or negative associations with mPFC FC. Additionally, to determine whether relationships between $\mathrm{mPFC} F C$ and pain rumination were specific to the patient group, we conducted a two-group with continuous covariate interaction analysis with $\mathrm{mPFC}$ FC maps from both control and patient groups inputted and pain rumination scores (mean from whole sample subtracted out) from each group entered as separate regressors. Contrasts were set up to identify voxels where the relationship between $\mathrm{mPFC}$ $\mathrm{FC}$ and pain rumination was greater in patients than in controls and vice versa. For these analyses, results were thresholded as done for the group differences analysis.

Additional analyses. We conducted additional analyses to confirm the validity and specificity of our results. Although we performed fMRI preprocessing procedures to reduce the impact of head motion on FC, head motion may influence FC even after standard preprocessing (Power et al., 2012; Van Dijk et al., 2012). We therefore calculated mean head motion (defined as mean absolute displacement of each volume compared with the previously acquired volume) within each subject and tested (1) whether controls and patients differed in terms of mean head motion (independent samples $t$ test), (2) whether group differences identified in $\mathrm{mPFC}-\mathrm{PCC} /$ precuneus $(\mathrm{PCu}) \mathrm{FC}$ were influenced by mean head motion (ANCOVA), and (3) whether the relationship between mPFC$\mathrm{PCC} / \mathrm{PCu} \mathrm{FC}$ and pain rumination in the TMD group was influenced by mean head motion (partial correlation).

Additionally, we examined whether our results were specific to FC of the $\mathrm{mPFC}$ with the DMN, or indicative of a general association of enhanced DMN FC in TMD associated with pain rumination. To do this, we performed an analysis that used another core node within the DMN as the seed region, the PCC. We defined the PCC as a $6 \mathrm{~mm}$-diameter sphere surrounding MNI coordinates ( $x y z=-8,-50,28)$ reported in our previous study from the contrast of brain activation during attention away from pain compared with attention toward pain (Kucyi et al., 2013), as done for the mPFC seed analysis (see Fig. 4). We used the same procedures as we did for the mPFC analysis to identify any possible group differences or associations with pain rumination for PCC FC.

\section{Results}

\section{Group differences in mPFC FC}

In both healthy control and TMD groups, there was statistically significant $\mathrm{FC}$ of the $\mathrm{MPFC}$ with the $\mathrm{PCC} / \mathrm{PCu}$, retrosplenial cortex (RSC) and medial temporal lobe (Fig. 1B; Table 1). The patients, but not controls, additionally showed significant FC of $\mathrm{mPFC}$ with the bilateral inferior parietal lobule (IPL) and lateral temporal cortex (Fig. 1B; Table 1). The group difference contrast revealed that the TMD patients had significantly enhanced $\mathrm{mPFC}$ FC with the PCC/PCu, RSC and areas within visual cortex compared with the control group (Fig. $1 C$; Table 1). There were no regions with significantly greater $\mathrm{MPFC} \mathrm{FC} \mathrm{in} \mathrm{controls} \mathrm{compared}$ with patients.

\section{Relationship between pain rumination and $\mathrm{mPFC} F \mathrm{~F}$}

In the TMD group, there were significant positive relationships between pain rumination and $\mathrm{MPFC}$ FC with clusters including peak voxels in the PCC, medial thalamus (mediodorsal nucleus), left PCC/PCu, medial thalamus (lateral dorsal nucleus), midbrain, anterior thalamus (ventral anterior nucleus), right $\mathrm{PCC} / \mathrm{PCu}$, and RSC, and voxels within the 
A mPFC FC positively related to pain rumination in TMD
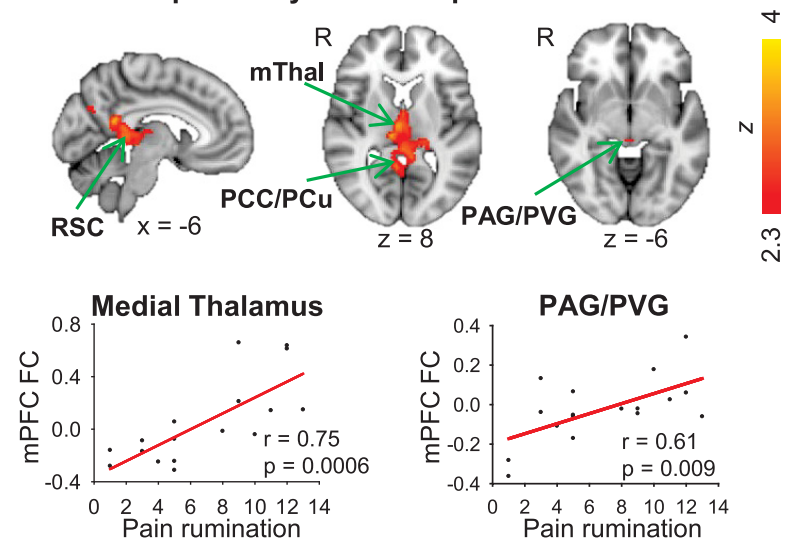

$B$ Specificity of brain-pain rumination relationships to TMD
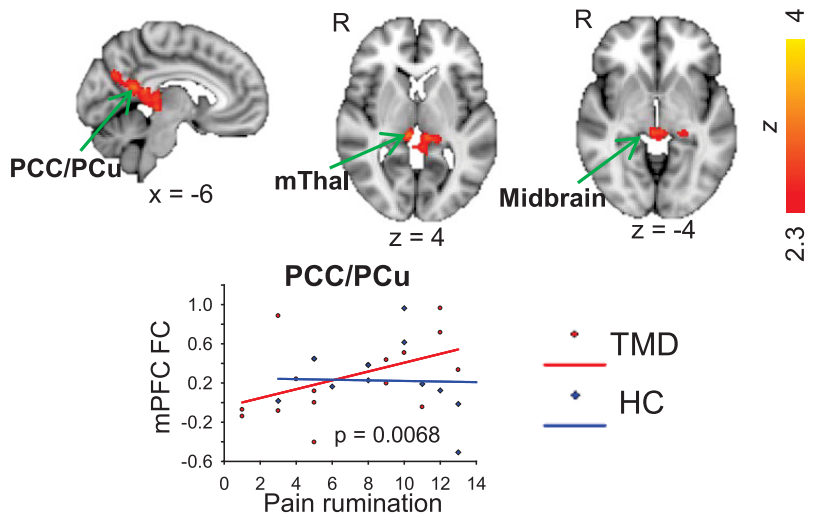

Figure 2. A, Relationship between resting state $\mathrm{MPFC} F \mathrm{C}$ and pain rumination. $A$, Brain regions exhibiting a significant positive relationship between pain rumination and $\mathrm{mPFC} F \mathrm{C}$ in TMD patients $(n=17$; FWE-corrected $Z>2.3$; cluster-based $p<0.05$ ). Correlation plots for $\mathrm{mPFC} F \mathrm{~F}$ with the $\mathrm{mThal}$ and PAG/PVG show pain rumination versus contrast of parameter estimate values extracted from the MNI coordinates listed in Table 2. $\boldsymbol{B}$, Results of group interaction analysis showing regions with stronger relationship between $\mathrm{mPFC} F \mathrm{C}$ and pain rumination in patients compared with control subjects, largely similar to regions shown in $\boldsymbol{A}$ (FWEcorrected $Z>2.3$; cluster-based $p<0.05$ ). Correlation plot shows pain rumination versus contrast of parameter estimate values extracted from $\mathrm{PCC} / \mathrm{PCu}$ peak coordinates for patients (red, circles) and controls (blue, diamonds). The interaction of pain rumination with group is significant in the regions shown ( $p=0.0068$ in peak voxel on the map). mThal, Medial thalamus.

periventricular gray (PVG)/PAG (Fig. 2A; Table 2). There were no regions with significant negative associations between pain rumination and $\mathrm{mPFC} F C$.

A group interaction analysis revealed that largely the same brain regions exhibited a significantly stronger relationship between MPFC FC and pain rumination in the TMD compared with control group. These regions included the PCC/PCu, RSC, medial thalamus and areas within the midbrain (Fig. 2B). There were no regions with a significantly stronger relationship between $\mathrm{mPFC}$ and pain rumination in controls compared with patients.

\section{Enhanced mPFC FC related to pain rumination in TMD patients}

To determine whether any brain regions exhibited both enhanced $\mathrm{mPFC}$ FC and significant correlation with pain rumination in TMD, we overlaid the significant $z$ maps from the two analyses on one another (Fig. 3). A cluster of 108 voxels in the $\mathrm{PCC} / \mathrm{PCu}$ was found to have overlap between the two $z$-maps
Table 2. Peak MNI coordinates for regions showing a significant positive correlation between $\mathrm{mPFC}$ functional connectivity and pain rumination in TMD patients (FWE-corrected at $Z>2.3$, cluster-based threshold of $p<0.05$ )

\begin{tabular}{|c|c|c|c|c|c|c|}
\hline \multirow[b]{2}{*}{ Region } & \multirow[b]{2}{*}{ Z-max } & \multicolumn{3}{|c|}{$\begin{array}{l}\text { Peak voxel } \\
\text { (MNI coordinates, mm) }\end{array}$} & \multirow[b]{2}{*}{$r$} & \multirow[b]{2}{*}{$p$} \\
\hline & & $x$ & $y$ & $z$ & & \\
\hline
\end{tabular}

Positive correlation between

MPFC FC and pain

rumination in TMD

PCC

$R$ medial thalamus

$\mathrm{LPCC} /$ precuneus

$R$ medial thalamus

Midbrain

L anterior thalamus

$\mathrm{RPCC} /$ precuneus

$R$ retrosplenial cortex

PVG/PAG

$\begin{array}{lrrrll}3.23 & 2 & -36 & 10 & 0.70 & 0.002 \\ 3.39 & 4 & -16 & 8 & 0.75 & 0.0006 \\ 3.54 & -6 & -52 & 18 & 0.77 & 0.0003 \\ 3.49 & 10 & -24 & 8 & 0.71 & 0.001 \\ 3.6 & 2 & -30 & -2 & 0.76 & 0.0004 \\ 3.7 & -2 & -6 & 0 & 0.75 & 0.0005 \\ 3.04 & 4 & -50 & 18 & 0.70 & 0.002 \\ 3.13 & 6 & -52 & 8 & 0.70 & 0.002 \\ 2.55 & 2 & -30 & -6 & 0.61 & 0.009\end{array}$

The image was thresholded at $Z>3$ to obtain peak coordinates (with exception of PVG/PAG where peak voxel at level of $z=-6$ is shown). Pearson's correlation coefficient values were extracted from voxels at listed coordinates.

(Fig. 3). This suggests that individuals who are higher in pain rumination most strongly drive enhanced $\mathrm{mPFC}-\mathrm{PCC} / \mathrm{PCu} \mathrm{FC}$ in TMD.

\section{Effects of head motion}

There was no significant group difference in mean head motion ( $p=0.31$; controls: mean $\pm \mathrm{SD}=0.16 \pm 0.086 \mathrm{~mm}$; patients: $0.13 \pm 0.070 \mathrm{~mm})$. When correcting for mean head motion, $\mathrm{mPFC}$ FC with the PCC/PCu (108 voxels as described above) remained enhanced in patients $(p=0.001)$. Furthermore, the positive correlation between pain rumination and $\mathrm{mPFC} \mathrm{FC}$ with the same PCC/PCu cluster in TMD remained significant when accounting for mean head motion $(r=0.75, p=0.001)$.

\section{PCC as an alternative seed region}

When the PCC was used as a seed region, canonical DMN maps were identified in both healthy control and patient groups (Fig. 4). In both groups, the PCC exhibited FC with the ventromedial/ dorsomedial PFC, lateral temporal cortex, and bilateral IPL. No group differences were identified in PCC FC with any brain region, and no significant relationships between pain rumination and PCC FC with any brain region were found within the TMD group (whole-brain FWE-corrected $Z<2.3$; cluster-based $p>$ $0.05)$.

\section{Discussion}

This study is the first to link DMN abnormalities in chronic pain with a pain-related cognitive factor. We demonstrate that patients with chronic pain exhibit abnormal resting state FC of the mPFC with areas of the DMN that reflects their individual degree of pain rumination, with individuals high in rumination having particularly enhanced mPFC-PCC/PCu FC. Additionally, we found that pain rumination in TMD relates to $\mathrm{MPFC} F \mathrm{FC}$ with the medial thalamus, an area associated with the affective and emotional aspects of pain (Treede et al., 2000), and with areas of the descending pain modulatory system (PAG/PVG) in patients. Together, our data suggest that in chronic pain, enhanced mPFC interactions with the DMN and the descending modulatory system play a prominent role in representing pain rumination.

The mPFC is well positioned, anatomically, to mediate DMN interactions with the descending pain modulatory system. Diffusion MRI studies of humans have revealed structural connectivity of the mPFC with the PAG (Hadjipavlou et al., 2006; Stein et al., 

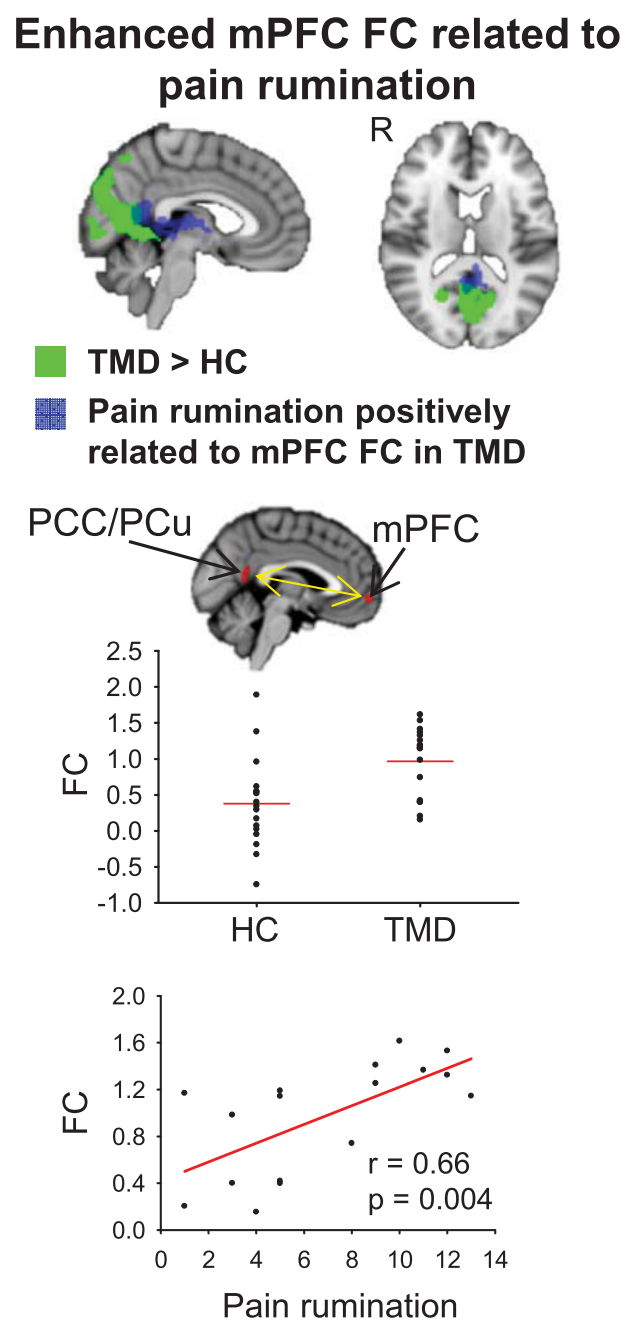

Figure 3. Top, Overlay of regions exhibiting significantly enhanced mPFC FC in TMD patients compared with healthy controls (green) and regions exhibiting a significant positive relationship between pain rumination and $\mathrm{mPFCFC}$ in TMD patients (blue). A cluster of 108 voxels in the PCC/PCu displayed overlap between the two statistical images. Middle, Mean contrast of parameter estimate values within the 108-voxel overlapping PCC/PCu cluster for $\mathrm{MPFCFC} \mathrm{plotted}$ for individuals in healthy control and patient groups. Bottom, Pain rumination scores within the TMD group plotted against mean contrast of parameter estimate values within the 108-voxel overlapping PCC/PCu cluster for mPFC FC.

2012; Kucyi et al., 2013) and PCC (Greicius et al., 2009). We recently showed that DMN activation and PAG-DMN FC increase during spontaneous attentional fluctuations away from pain (Kucyi et al., 2013). Furthermore, we found associations of mPFC-PAG structural and resting FC with individual differences in the tendency to attend to pain (Kucyi et al., 2013). In chronic pain, rumination in daily life likely affects interactions between similar brain regions and is represented in resting state $\mathrm{FC}$ as identified here.

Structural abnormalities of the mPFC have been identified in multiple chronic pain populations (May, 2011) including TMD (Moayedi et al., 2011). Abnormal task-related DMN deactivation has been found in chronic back pain (Baliki et al., 2008) and TMD (Weissman-Fogel et al., 2011). Additionally, abnormalities in resting state FC of the DMN have been found in fibromyalgia (Napadow et al., 2010), chronic back pain (Baliki et al., 2011; Loggia et al., 2013), migraine (Xue et al., 2012), and complex regional pain syndrome (Bolwerk et al., 2013). The reported brain regions exhibiting changes in DMN FC are varied across

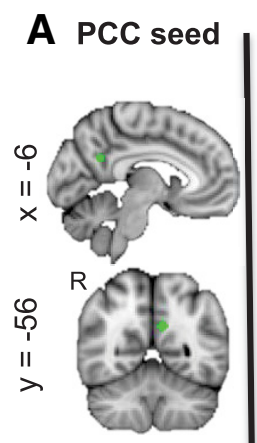

B PCC resting state FC

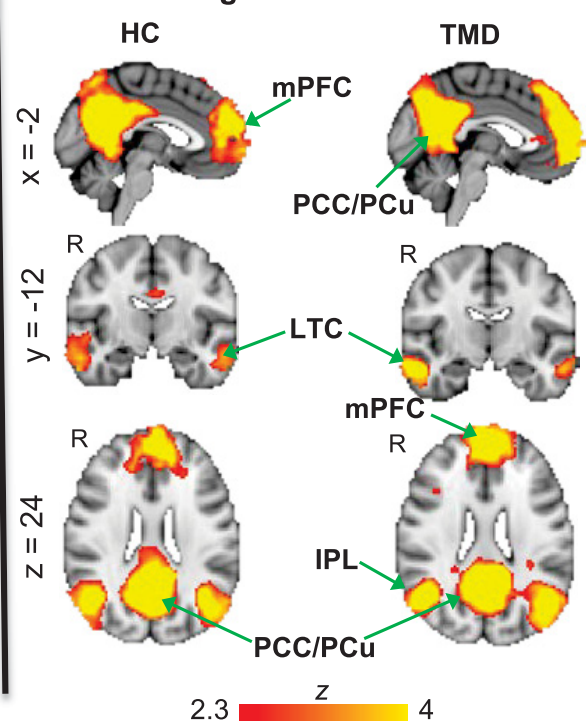

Figure 4. PCC FC in healthy controls and TMD patients. $A$, Location of the $P C C$ seed region in standard MNI152 space. $\boldsymbol{B}$, Brain regions exhibiting resting state $\mathrm{FC}$ with the $\mathrm{PCC}$ in healthy controls $(n=17)$ and TMD patients $(n=17)$. All statistical images are thresholded at wholebrain FWE-corrected $Z>2.3$; cluster-based $p<0.05$. IPL, Inferior parietal lobule; LTC, lateral temporal cortex.

studies, possibly due to differences among chronic pain conditions. In some cases, DMN FC with pain-related brain regions (e.g., insula) has been shown to correlate with clinical pain intensity (Napadow et al., 2010; Loggia et al., 2013). Longitudinal studies have revealed that $\mathrm{FC}$ of the $\mathrm{mPFC}$ with limbic regions during subacute back pain predicts the development of chronic pain (Baliki et al., 2012) and that improvements in clinical pain in fibromyalgia are associated with changes in DMN-insula FC ( Napadow et al., 2012). Our study builds on this growing literature of $\mathrm{mPFC} / \mathrm{DMN}$ abnormalities in chronic pain by showing for the first time aberrant resting state DMN FC in TMD and an association between resting state DMN FC and pain rumination.

Interestingly, our finding of enhanced mPFC-DMN FC in patients with chronic pain is in line with results from an arterial spin-labeling (ASL) study in chronic low back pain patients (Loggia et al., 2013). However, in that study, an independent components analysis coupled with dual regression revealed a negative correlation between mPFC-DMN FC and clinical pain intensity. Loggia et al. (2013) suggested that enhanced FC of this circuitry could be a compensatory mechanism wherein FC may be enhanced in patients to lower their pain levels. Here, our BOLD fMRI and seed-based connectivity analysis showed a positive correlation between $\mathrm{mPFC}-\mathrm{DMN}$ FC and pain rumination, and notably pain rumination was not related to clinical pain intensity. Furthermore, we correlated average pain intensity in TMD patients to mPFC FC with the 108-voxel PCC/PCu cluster shown in Figure 3 and found no significant relationship $(r=-0.23$; $p=$ $0.37)$. Therefore, although enhanced mPFC-DMN FC may be generalized to chronic pain arising from different etiologies, the relationship of FC with clinical pain intensity may be dependent upon specific type of pain, modality of acquired data (ASL vs BOLD), and/or analysis approach.

In addition to abnormalities in $\mathrm{MPFC} F C$ with the DMN, we found enhanced mPFC FC with visual areas within occipital cortex in TMD patients relative to healthy controls. We are not aware of any other studies showing abnormal FC of the DMN with 
visual regions in chronic pain. However, there is some evidence for aberrant resting state FC of visual regions with areas involved in pain affect in patients with chronic migraine (Schwedt et al., 2013). As we found no significant correlations between pain rumination and $\mathrm{mPFC} F \mathrm{~F}$ with visual areas, the behavioral significance of the enhanced FC is a topic of future endeavor.

Beyond studies of chronic pain, resting state DMN FC has been shown to be disrupted in psychiatric populations (WhitfieldGabrieli and Ford, 2012). For example, in major depressive disorder, enhanced mPFC-DMN FC has been associated with rumination about negative events (Zhu et al., 2012), similar to the association we found here with pain rumination. This raises the possibility of common effects on the DMN due to repetitive and distressing thoughts and feelings. One possibility is that rumination sustains the communication between the MPFC and DMN. This can be thought of as a system that is "stuck in a rut" with little flexibility for interactions with other networks, leading to enhanced overall mPFC-DMN FC. Future studies could identify changes in short time-scale network dynamics related to rumination with newer approaches such as those available with longer fMRI scan durations (Hutchison et al., 2013) or with higher temporal resolution such as that offered by magnetoencephalography.

A link between pain rumination and $\mathrm{mPFC}$ connectivity with the PVG/PAG may be unique to people with chronic pain, possibly reflecting individual differences in pain modulation. Notably, although a role of the PAG in descending pain inhibition is well established (Millan, 2002), there is also evidence from animal studies for pronociceptive effects of the PAG under certain contexts such as stress (Fields, 2004; Lovick, 2008). As we found positive correlations of $\mathrm{MPFC}$ FC with pain rumination for both the mediodorsal thalamus (a likely pronociceptive region) and PAG, it is plausible that the $\mathrm{mPFC} F C$ with the PAG reflects a pronociceptive pathway coupled with the thalamus. However, it remains unknown how ongoing PAG activity in human chronic pain populations represents antinociceptive versus pronociceptive functions. Human imaging studies of acute pain in healthy individuals largely focus on an antinociceptive function of the PAG (Tracey and Mantyh, 2007), but activation of the PAG during pain anticipation may promote hyperalgesia (Fairhurst et al., 2007). To explore the functional significance of mPFC-PAG FC and its link with pain rumination in more detail, future studies could employ continuous online ratings of pain and perceptrelated fMRI (Davis et al., 2002; Kwan et al., 2005) in patients with chronic pain.

Given that the mediodorsal thalamus plays a role in the affective dimension of pain (Treede et al., 2000), the link between pain rumination and the mPFC-mediodorsal thalamus FC could reflect persistent attempts to regulate pain. Altered mPFCmediodorsal thalamus FC has been shown to be related to cognitive impairments in rats with inflammatory pain (Cardoso-Cruz et al., 2013). Pain rumination may thus be involved with such cognitive deficits and their association with affect, leading to enhanced mPFC-mediodorsal thalamus FC in chronic pain as identified here. It is notable that the healthy controls did not show a significant correlation between pain rumination and mPFC-thalamus/PAG FC. This suggests that the degree to which chronic pain states alter normal function of these circuits depends on how much a given patient ruminates.

\section{References}

Andrews-Hanna JR, Reidler JS, Sepulcre J, Poulin R, Buckner RL (2010) Functional-anatomic fractionation of the brain's default network. Neuron 65:550-562. CrossRef Medline
Baliki MN, Geha PY, Apkarian AV, Chialvo DR (2008) Beyond feeling: chronic pain hurts the brain, disrupting the default-mode network dynamics. J Neurosci 28:1398-1403. CrossRef Medline

Baliki MN, Baria AT, Apkarian AV (2011) The cortical rhythms of chronic back pain. J Neurosci 31:13981-13990. CrossRef Medline

Baliki MN, Petre B, Torbey S, Herrmann KM, Huang L, Schnitzer TJ, Fields HL, Apkarian AV (2012) Corticostriatal functional connectivity predicts transition to chronic back pain. Nat Neurosci 15:1117-1119. CrossRef Medline

Behzadi Y, Restom K, Liau J, Liu TT (2007) A component based noise correction method (CompCor) for BOLD and perfusion based fMRI. Neuroimage 37:90-101. CrossRef Medline

Bolwerk A, Seifert F, Maihöfner C (2013) Altered resting-state functional connectivity in complex regional pain syndrome. J Pain 14:1107-1115. CrossRef Medline

Buenaver LF, Quartana PJ, Grace EG, Sarlani E, Simango M, Edwards RR, Haythornthwaite JA, Smith MT (2012) Evidence for indirect effects of pain catastrophizing on clinical pain among myofascial temporomandibular disorder participants: the mediating role of sleep disturbance. Pain 153:1159-1166. CrossRef Medline

Cardoso-Cruz H, Sousa M, Vieira JB, Lima D, Galhardo V (2013) Prefrontal cortex and mediodorsal thalamus reduced connectivity is associated with spatial working memory impairment in rats with inflammatory pain. Pain 154:2397-2406. CrossRef Medline

Chai XJ, Castañón AN, Ongür D, Whitfield-Gabrieli S (2012) Anticorrelations in resting state networks without global signal regression. Neuroimage 59:1420-1428. CrossRef Medline

Christoff K, Gordon AM, Smallwood J, Smith R, Schooler JW (2009) Experience sampling during fMRI reveals default network and executive system contributions to mind wandering. Proc Natl Acad Sci U S A 106: 8719-8724. CrossRef Medline

Davis KD, Pope GE, Crawley AP, Mikulis DJ (2002) Neural correlates of prickle sensation: a percept-related fMRI study. Nat Neurosci 5:11211122. CrossRef Medline

Desouza DD, Moayedi M, Chen DQ, Davis KD, Hodaie M (2013) Sensorimotor and pain modulation brain abnormalities in trigeminal neuralgia: A paroxysmal, sensory-triggered neuropathic pain. PLoS One 8:e66340. CrossRef Medline

Fairhurst M, Wiech K, Dunckley P, Tracey I (2007) Anticipatory brainstem activity predicts neural processing of pain in humans. Pain 128:101-110. CrossRef Medline

Fields H (2004) State-dependent opioid control of pain. Nat Rev Neurosci 5:565-575. CrossRef Medline

Greicius MD, Krasnow B, Reiss AL, Menon V (2003) Functional connectivity in the resting brain: a network analysis of the default mode hypothesis. Proc Natl Acad Sci U S A 100:253-258. CrossRef Medline

Greicius MD, Supekar K, Menon V, Dougherty RF (2009) Resting-state functional connectivity reflects structural connectivity in the default mode network. Cereb Cortex 19:72-78. CrossRef Medline

Hadjipavlou G, Dunckley P, Behrens TE, Tracey I (2006) Determining anatomical connectivities between cortical and brainstem pain processing regions in humans: a diffusion tensor imaging study in healthy controls. Pain 123:169-178. CrossRef Medline

Hutchison RM, Womelsdorf T, Allen EA, Bandettini PA, Calhoun VD, Corbetta M, Della Penna S, Duyn JH, Glover GH, Gonzalez-Castillo J, Handwerker DA, Keilholz S, Kiviniemi V, Leopold DA, de Pasquale F, Sporns O, Walter M, Chang C (2013) Dynamic functional connectivity: promise, issues, and interpretations. Neuroimage 80:360-378. CrossRef Medline

Jenkinson M, Beckmann CF, Behrens TE, Woolrich MW, Smith SM (2012) FSL. Neuroimage 62:782-790. CrossRef Medline

Kross E, Davidson M, Weber J, Ochsner K (2009) Coping with emotions past: the neural bases of regulating affect associated with negative autobiographical memories. Biol Psychiatry 65:361-366. CrossRef Medline

Kucyi A, Hodaie M, Davis KD (2012) Lateralization in intrinsic functional connectivity of the temporoparietal junction with salience- and attentionrelated brain networks. J Neurophysiol 108:3382-3392. CrossRef Medline

Kucyi A, Salomons TV, Davis KD (2013) Mind wandering away from pain dynamically engages antinociceptive and default mode brain networks. Proc Natl Acad Sci U S A 110:18692-18697. CrossRef Medline

Kwan CL, Diamant NE, Pope G, Mikula K, Mikulis DJ, Davis KD (2005) 
Abnormal forebrain activity in functional bowel disorder patients with chronic pain. Neurology 65:1268-1277. CrossRef Medline

Loggia ML, Kim J, Gollub RL, Vangel MG, Kirsch I, Kong J, Wasan AD, Napadow V (2013) Default mode network connectivity encodes clinical pain: an arterial spin labeling study. Pain 154:24-33. CrossRef Medline

Lovick TA (2008) Pro-nociceptive action of cholecystokinin in the periaqueductal grey: a role in neuropathic and anxiety-induced hyperalgesic states. Neurosci Biobehav Rev 32:852-862. CrossRef Medline

Mainero C, Boshyan J, Hadjikhani N (2011) Altered functional magnetic resonance imaging resting-state connectivity in periaqueductal gray networks in migraine. Ann Neurol 70:838-845. CrossRef Medline

May A (2011) Structural brain imaging: a window into chronic pain. Neuroscientist 17:209-220. CrossRef Medline

Millan MJ (2002) Descending control of pain. Prog Neurobiol 66:355-474. CrossRef Medline

Moayedi M, Weissman-Fogel I, Crawley AP, Goldberg MB, Freeman BV, Tenenbaum HC, Davis KD (2011) Contribution of chronic pain and neuroticism to abnormal forebrain gray matter in patients with temporomandibular disorder. Neuroimage 55:277-286. CrossRef Medline

Moayedi M, Weissman-Fogel I, Salomons TV, Crawley AP, Goldberg MB, Freeman BV, Tenenbaum HC, Davis KD (2012a) Abnormal gray matter aging in chronic pain patients. Brain Res 1456:82-93. CrossRef Medline

Moayedi M, Weissman-Fogel I, Salomons TV, Crawley AP, Goldberg MB, Freeman BV, Tenenbaum HC, Davis KD (2012b) White matter brain and trigeminal nerve abnormalities in temporomandibular disorder. Pain 153:1467-1477. CrossRef Medline

Napadow V, LaCount L, Park K, As-Sanie S, Clauw DJ, Harris RE (2010) Intrinsic brain connectivity in fibromyalgia is associated with chronic pain intensity. Arthritis Rheum 62:2545-2555. CrossRef Medline

Napadow V, Kim J, Clauw DJ, Harris RE (2012) Decreased intrinsic brain connectivity is associated with reduced clinical pain in fibromyalgia. Arthritis Rheum 64:2398-2403. CrossRef Medline

Nolen-Hoeksema S (2000) The role of rumination in depressive disorders and mixed anxiety/depressive symptoms. J Abnorm Psychol 109:504511. CrossRef Medline

Power JD, Barnes KA, Snyder AZ, Schlaggar BL, Petersen SE (2012) Spurious but systematic correlations in functional connectivity MRI networks arise from subject motion. Neuroimage 59:2142-2154. CrossRef Medline

Raichle ME, Snyder AZ (2007) A default mode of brain function: a brief history of an evolving idea. Neuroimage 37:1083-1090; discussion 10971099. CrossRef Medline

Rocca MA, Ceccarelli A, Falini A, Colombo B, Tortorella P, Bernasconi L, Comi G, Scotti G, Filippi M (2006) Brain gray matter changes in migraine patients with T2-visible lesions: a 3-T MRI study. Stroke 37:17651770. CrossRef Medline

Salomons TV, Moayedi M, Weissman-Fogel I, Goldberg MB, Freeman BV, Tenenbaum HC, Davis KD (2012) Perceived helplessness is associated with individual differences in the central motor output system. Eur J Neurosci 35:1481-1487. CrossRef Medline
Sansone RA, Sansone LA (2012) Rumination: relationships with physical health. Innov Clin Neurosci 9:29-34. Medline

Schwedt TJ, Schlaggar BL, Mar S, Nolan T, Coalson RS, Nardos B, Benzinger T, Larson-Prior LJ (2013) Atypical resting-state functional connectivity of affective pain regions in chronic migraine. Headache 53:737-751. CrossRef Medline

Stein N, Sprenger C, Scholz J, Wiech K, Bingel U (2012) White matter integrity of the descending pain modulatory system is associated with interindividual differences in placebo analgesia. Pain 153:2210-2217. CrossRef Medline

Sullivan MJ, Bishop SR, Pivik J (1995) The pain catastrophizing scale: development and validation. Psychol Assess 7:524-532. CrossRef

Sullivan MJ, Sullivan ME, Adams HM (2002) Stage of chronicity and cognitive correlates of pain-related disability. Cogn Behav Ther 31:111-118. CrossRef

Tracey I, Mantyh PW (2007) The cerebral signature for pain perception and its modulation. Neuron 55:377-391. CrossRef Medline

Treede RD, Apkarian AV, Bromm B, Greenspan JD, Lenz FA (2000) Cortical representation of pain: functional characterization of nociceptive areas near the lateral sulcus. Pain 87:113-119. CrossRef Medline

Van Damme S, Crombez G, Bijttebier P, Goubert L, Van Houdenhove B (2002) A confirmatory factor analysis of the Pain Catastrophizing Scale: invariant factor structure across clinical and non-clinical populations. Pain 96:319-324. CrossRef Medline

Van Dijk KR, Sabuncu MR, Buckner RL (2012) The influence of head motion on intrinsic functional connectivity MRI. Neuroimage 59:431-438. CrossRef Medline

Weissman DH, Roberts KC, Visscher KM, Woldorff MG (2006) The neural bases of momentary lapses in attention. Nat Neurosci 9:971-978. CrossRef Medline

Weissman-Fogel I, Moayedi M, Tenenbaum HC, Goldberg MB, Freeman BV, Davis KD (2011) Abnormal cortical activity in patients with temporomandibular disorder evoked by cognitive and emotional tasks. Pain 152: 384-396. CrossRef Medline

Whitfield-Gabrieli S, Ford JM (2012) Default mode network activity and connectivity in psychopathology. Annu Rev Clin Psychol 8:49-76. CrossRef Medline

Worsley KJ, Liao CH, Aston J, Petre V, Duncan GH, Morales F, Evans AC (2002) A general statistical analysis for fMRI data. Neuroimage 15:1-15. CrossRef Medline

Xue T, Yuan K, Zhao L, Yu D, Dong T, Cheng P, von Deneen KM, Qin W, Tian J (2012) Intrinsic brain network abnormalities in migraines without aura revealed in resting-state fMRI. PLoS One 7:e52927. CrossRef Medline

Zhu X, Wang X, Xiao J, Liao J, Zhong M, Wang W, Yao S (2012) Evidence of a dissociation pattern in resting-state default mode network connectivity in first-episode, treatment-naive major depression patients. Biol Psychiatry 71:611-617. CrossRef Medline 